\title{
La sélection des spermatozoïdes à fort grossissement permet-elle une diminution de la fréquence des aneuploïdies spermatiques?
}

\author{
Mohamed Hassen CHELLI1, Martine ALBERT1,2, Denise MOLINA GOMEZ1,2, \\ Ibrahim HAMMOUD1,2, Jaqueline SELVA1,2, François VIALARD1,2
}
1 Service d'Histologie-Embryologie-Cytogénétique, Biologie de la Reproduction et Génétique Médicale, CHI Poissy-Saint Germain en Laye ; 2 UFR Paris Île de France Ouest, Université Versailles - Saint Quentin en Yvelines

\begin{abstract}
RESUME
L'infertilité masculine sévère peut se retrouver chez 2 catégories d'hommes. Les hommes infertiles à caryotype anormal, qui représentent 2 à $14 \%$ des sujets infertiles, peuvent produire des spermatozoïdes porteurs du déséquilibre chromosomique lié au remaniement initial du patient entraînant un risque variable de transmission de cette anomalie à leur conceptus. La deuxième catégorie est celle des hommes infertiles à caryotype normal chez qui Il a été décrit un taux d'aneuploïdie spermatique augmenté lorsqu'ils présentaient une oligo et ou une asthénozoospermie sévère et/ou lorsqu'ils étaient issus de couples en échec d'implantation. Le traitement classique en Assistance Médicale à la Procréation pour ses 2 catégories étant l'ICSI en dépit du risque chromosomique accru évident. Se pose alors la question de savoir s'il est possible de reconnaître morphologiquement les spermatozoïdes au contenu chromosomique anormal au cours de l'ICSI.
\end{abstract}

Malheureusement, il n'a pas été mis en évidence de relation entre la morphologie des spermatozoïdes au grossissement x200 de l'ICSI et leur contenu chromosomique. Néanmoins depuis la fin des années 90, l'équipe de Bartoov a mis au point une méthode d'observation fine de la morphologie spermatique, dénommée MSOME, qui propose d'observer les spermatozoïdes avec un grossissement allant jusqu'à $x 12250$. Cette technique étant proposée dans les cas d'échecs répétés d'ICSI, l'amélioration des taux d'implantation ainsi que des taux de grossesse est-elle due à une meilleure sélection du contenu chromosomique du spermatozoïde à injecter ?

L'objectif de ce travail a donc été d'évaluer l'intérêt du MSOME dans la sélection des spermatozoïdes euploïdes (contenu chromosomique normal). Pour cela, nous avons choisi de travailler sur 2 groupes de patients connus pour leur taux d'aneuploïdie spermatique augmenté.
Le groupe 1, constitué de 2 patients à caryotype normal, présentant un syndrome de macrocéphalie spermatique avec plus de $99 \%$ de spermatozoïdes aneuploïdes.

Les groupes 2, constitués de 11 patients à caryotype anormal, 6 patients porteurs de translocations réciproques et 5 patients porteurs de translocations robertsonniennes.

Le but de l'étude était de comparer les résultats de la FISH sur spermatozoïdes après sélection au grossissement ICSI et MSOME par rapport au sperme entier. Les particularités de notre protocole ont été : (1) tous les spermatozoïdes sélectionnés en MSOME étaient des "top spermatozoïdes" ; (2) de fixer les spermatozoïdes sélectionnés ; (3) valider la lecture en FISH sur les cellules sélectionnés par deux lecteurs différents.

L'analyse en FISH des chromosomes $X, Y$ et 18 chez les patients présentant la macrocéphalie spermatique a montré que la sélection en MSOME élimine les spermatozoïdes polyploïdes et diploïdes, mais les 6 spermatozoïdes sélectionnés sont tous haploïdes aneuploïdes. Pour les 5 sujets porteurs de translocations robertsonniennes, les résultats de l'analyse en FISH ont montré paradoxalement une diminution significative du taux de spermatozoïdes euploïdes en sélection MSOME par rapport au sperme entier. Concernant les 6 patients porteurs de translocations réciproques, les résultats globaux de l'analyse en FISH des différentes translocations réciproques étaient homogènes entre les patients et le taux d'aneuploödie global n'était pas différent entre le sperme entier et les 2 méthodes de

\section{Correspondance :}

Dr Mohamed Hassen CHELLI - Service d'Histologie-

Embryologie-Cytogénétique, Biologie de la Reproduction et Génétique Médicale. CHI Poissy-Saint Germain en Laye,

78303 POISSY cedex - Email

mohamedhassenchelli@yahoo.fr 
sélection. Par contre, nous avons remarqué une diminution significative de la fréquence des ségrégations adjacente 1 et 2 entre le sperme entier et la sélection en MSOME, accompagnée d'une augmentation significative de la fréquence de la ségrégation 3:1. Les ségrégations (adjacente 1 et 2) qui modifient la structure des chromosomes pourraient entraîner des modifications morphologiques visibles en MSOME et seraient donc éliminées. L'hypothèse d'une modulation de l'efficacité de l'apoptose spermatique en fonction de la morphologie et du contenu chromosomique du spermatozoïde est à discuter.

En conclusion, le MSOME semble permettre d'éliminer les spermatozoïdes di ou polyploïdes, mais ne garantit pas le contenu chromosomique haploïde des "top" spermatozoïdes sélectionnés. De plus, les résultats que nous avons obtenus sur une petite série de patients suggèrent que le MSOME pourrait éliminer certaines anomalies chromosomiques qui altèreraient la structure nucléaire spermatique.

Mots clefs : spermatozoïde, morphologie, MSOME, macrocéphalie, translocation, FISH

\section{INTRODUCTION}

L'ICSI (Intra Cytoplasmic Sperm Injection) a transformé les chances de procréation des patients présentant un déficit important de la spermatogenèse [14]. Cette technique d'Assistance Médicale à la Procréation (AMP) est largement utilisée dans les infertilités d'origine masculine [37, 40], qu'il s'agisse d'aitérations spermatiques qualitatives comme la tératozoospermie et l'asthénozoospermie ou quantitatives comme l'oligozoospermie [11, 29, 30]. Or, dans les cas d'infertilité masculine sévère à caryotype normal, une augmentation de la prévalence des aneuploïdies concernant plus particulièrement les anomalies des gonosomes [22], a été rapportée chez les foetus conçus après ICSI [38]. Ce résultat semble dû à une augmentation du taux d'aneuploïdie dans le sperme des patients infertiles à caryotype normal par rapport à celui de témoins $[10,27,32]$. La concentration spermatique est à l'évidence un indicateur du risque chromosomique avec une augmentation du risque liée à la sévérité de l'oligozoospermie [25, 34].

Concernant la morphologie spermatique, si l'on excepte les tératospermies syndromiques majeures touchant la tête spermatique telles que la macrocéphalie ou la globozoospermie, aucune relation entre la morphologie du sperme évaluée par le spermocytogramme et les taux de succès en ICSI n'a été mise en évidence. Les taux d'implantation et de grossesse clinique sont cependant diminués dans les cas où seuls des spermatozoïdes morphologiquement anormaux sont disponibles pour la micro-injection [13]. Un taux élevé de macrocéphalie est un des marqueurs phénotypiques à haut risque d'anomalies chromosomiques, les spermatozoïdes macrocéphales étant tous polyploïdes. Le recours au Diagnostic Pré-Implantatoire (DPI) serait d'autant plus justifié que les spermatozoïdes de taille apparemment normale, présents dans ces spermes à fort taux de macrocéphalie (90-95\%), sont le plus souvent aneuploïdes [16, 20].

D'autres anomalies morphologiques semblent également être liées à un taux élevé d'aneuploïdies chromosomiques spermatiques comme certaines dysmorphies flagellaires [30]. Les tératozoospermies majeures sont d'ailleurs, dans leur ensemble, considérées comme des indications au DPI par certains auteurs [33]. De plus, des situations cliniques comme l'échec d'implantation (plus de 10 embryons transférés sans grossesse) et les fausses couches à répétition chez les couples à caryotype normal, sont parfois liées à une augmentation du risque chromosomique [27], souvent ovocytaire mais aussi spermatique, l'aspect « normal » d'un spermatozoïde lors d'une ICSI classique ne préjugeant pas de la normalité de son contenu chromosomique.

Enfin, l'étude chromosomique des hommes infertiles a révélé que 2 à $14 \%$ de ces sujets avaient un caryotype constitutionnel anormal [34]. Ces anomalies peuvent d'une part, causer des altérations des paramètres spermatiques et d'autre part, conduire à la production de spermatozoïdes porteurs de déséquilibres chromosomiques. L'ICSI ne permet pas d'exclure les spermatozoïdes porteurs de ces anomalies déséquilibrées lors de la fécondation [10]. Elle est donc mise en cause dans la transmission de ces aneuploïdies au conceptus [10,34, 40]

Au total, on est donc amené en ICSI à injecter un spermatozoïde à risque chromosomique accru dans différentes situations : patients à caryotype normal mais avec une numération spermatique très abaissée, certaines tératozoospermies et patients à caryotype anormal.

Bartoov et al. [2, 3] ont développé une méthode permettant une évaluation à très fort grossissement de la morphologie des spermatozoïdes mobiles en temps réel ou MSOME (Motile Sperm Organelle Morphology Examination). La sélection du spermatozoïde par la morphologie fine, notamment nucléaire, évaluée à un grossissement supérieur à x6000 semble alors constituer un facteur important de succès après ICSI. Cette méthode appliquée à la sélection de spermatozoïdes mobiles présentant une morphologie et un contenu nucléaire normaux pour l'ICSI prend le nom d'IMSI (Intracytoplasmic Morphologically Selected sperm injection).

Les premiers résultats publiés sont très encourageants puisqu'ils font état d'une augmentation significative des taux d'implantation et de grossesse chez des couples ayant eu deux échecs successifs en ICSI, avec une diminution du taux de fausse couche spontanée lorsque la micro-injection se fait avec des spermatozoïdes morphologiquement sélectionnés en MSOME. En cas d'échec d'implantation préalable (plus de 2 tentatives sans grossesse), une augmentation des taux d'implantation et de grossesse a été rapportée en utilisant cette approche [18].

Sachant qu'environ un embryon humain sur deux est porteur d'une anomalie chromosomique [17], nous nous sommes demandés si la sélection morphologique des spermatozoïdes par MSOME agirait sur les taux de succès en IMSI par le biais d'une sélection des anomalies chromosomiques. Cette 
hypothèse expliquerait en particulier l'augmentation des taux de grossesse clinique observée dans des indications comme les échecs répétés d'implantation et la chute du taux de fausses couches spontanées.

Nous avons donc choisi d'évaluer l'intérêt de la sélection par MSOME en vue d'une IMSI dans la prise en charge de couples à risque d'aneuploïdie augmenté et de savoir si cette technique permettrait dans ces situations de sélectionner des spermatozoïdes ayant de plus grandes chances d'être euploïdes. Cette étude a concerné (groupe 1) des couples infertiles à caryotype normal avec plus de $5 \%$ d'aneuploïdie spermatique concernant les chromosomes $X$, $Y$ et 18 (valeur normale chez des patients fertiles $<1 \%$ ) et (groupe 2 ) des couples dont le conjoint présente un caryotype anormal.

Pour chaque patient, l'analyse en FISH (Fluorescent In Situ Hybridization) a été réalisée sur les spermatozoïdes du sperme entier, les spermatozoïdes sélectionnés à l'objectif d'ICSI (x200) et les spermatozoïdes sélectionnés au MSOME (x6000). L'analyse par FISH a concerné, pour tous les patients, les chromosomes $X$ et $Y$ qui sont les plus fréquemment impliqués dans les aneuploïdies spermatiques et le chromosome 18 représentatif des autosomes. Pour les patients porteurs d'une translocation (groupe 2), les déséquilibres liés à la translocation ont été également évalués avec les sondes spécifiques de chaque chromosome impliqué dans la translocation.

\section{MATERIEL ET METHODES}

\section{Matériel}

Cette étude s'est déroulée entre novembre 2006 et juillet 2007 dans le Service d'Histologie, Embryologie, Biologie de la Reproduction, Cytogénétique et Génétique Médicale au Centre Hospitalier Intercommunal de Poissy Saint-Germainen-Laye. Deux groupes de patients nous ont intéressé :

- Groupe 1 ( $n=2$, Tableau 1) : Patients infertiles à caryotype normal, ayant une macrocéphalie spermatique à l'origine d'une augmentation du taux d'aneuploïdie spermatique. Le patient 1 est issu d'un couple en échec d'implantation (5 tentatives d'ICSI dans différents centres, 12 embryons transférés au total, sans grossesse). Le spermogramme de contrôle, a confirmé un syndrome de macrocéphalie spermatique. Le patient 2 s'est présenté à notre centre pour confirmation d'un syndrome de macrocéphalie spermatique suite à une ICSI tentée dans un autre centre et au cours de laquelle 8 ovocytes avaient été ponctionnés et aucun injecté par défaut de spermatozoïde injectable. Nous avons confirmé la macrocéphalie au spermocytogramme.

- Groupe $2(n=11$, Tableau 2) : Patients à caryotype anormal, recrutés au cours de la consultation de génétique, soit pré ICSI pour les patients $3,4,5,9,10,11$, et 13 , soit pour le DPN (Diagnostic Pré-Natal) pour les patients 6,7 , 8 , et 12 . Au total, nous avons étudié 6 cas de translocations réciproques équilibrées, et 5 cas de translocations robertsonniennes.

Nous avons considéré comme spermocytogramme de référence l'évaluation de la morphologie spermatique réalisée sur sperme entier selon la méthode de David modifiée [1, 12] couplée aux critères stricts de Kruger [21]. Notre valeur de référence pour le pourcentage de formes typiques était de $15 \%$.

Dans le cadre de la consultation génétique pré-ICSI, les patients ont signé un consentement pour la réalisation d'un caryotype constitutionnel. Le protocole de sélection des spermatozoïdes au MSOME, associé à l'étude du contenu chromosomique par FISH sur sperme frais et après sélection, a été également soumis au consentement écrit des patients. Ce travail a été réalisé avec le soutien financier de l'Agence de la Biomédecine.

\section{Méthodes}

Notre avons mené notre étude selon le schéma méthodologique qui suit (Figure 1).

\section{a) Préparation du sperme}

Pour chaque patient, l'échantillon de sperme a été divisé en 2 fractions après liquéfaction $\left(30-45\right.$ min à $\left.37^{\circ} \mathrm{C}\right)$ :

- FISH sur sperme entier : le sperme a été centrifugé à $600 \mathrm{~g}$ pendant $10 \mathrm{~min}$. Après élimination du liquide séminal, le culot a été lavé 2 fois dans $1,5 \mathrm{ml}$ d'eau distillée. Les spermatozoïdes ont été ensuite fixés dans du Carnoy acétique ( 3 volumes de méthanol pour 1 volume d'acide acétique) et le prélèvement a été alors conservé à $+4^{\circ} \mathrm{C}$ pour les études de FISH ultérieures. Un minimum de 500 spermatozoïdes lus par lame était requis.

- FISH sur spermatozoïdes sélectionnés : le sperme a été mis à migrer sur gradient bicouche PureSperm $100 \circledast$ (Nidacon, JCD France) (45\%-90\%) à $300 \mathrm{~g}$ pendant 20 min. La fraction $90 \%$ a été ensuite lavée avec $2 \mathrm{ml}$ de milieu de culture Universal IVF Medium® (MediCult France) à $600 \mathrm{~g}$ pendant $10 \mathrm{~min}$. Le culot obtenu a été dilué dans un volume approprié de milieu de culture avant sélection. Nous avons essayé de sélectionner 100 spermatozoïdes en ICSI et 100 spermatozoïdes en MSOME pour chaque patient dans un délai maximal de 3 heures.

\section{b) Technique d'observation et d'analyse morphologique des spermatozoïdes sélectionnés au fort grossissement (MSOME)}

Un échantillon de $2 \mu$ de sperme migré a été dilué dans une micro-goutte de $4 \mu$ le polyvinyl pyrrolidone (10\% PVP in flushing medium, FertiPro N.V., Belgium) puis placé dans une boîte WillCo-dish $\otimes$ (WillCoWells B.V. Amsterdam, The Netherlands) à fond en verre d'épaisseur $0,17 \mathrm{~mm}$, afin de permettre l'observation sous huile de paraffine stérile. L'examen a été réalisé à l'aide d'un microscope inversé Nikon ${ }^{\circledR}$ équipé d'un objectif $\times 100$ Nomarsky permettant à l'immersion un grossissement final de $x 1500$ aux oculaires. Le système optique comprend ensuite une caméra numérique couleur Sony ${ }^{\circledR}$ permettant l'acquisition de 50 images/sec ainsi qu'un écran JVC ${ }^{\circledR}$ de 15 ".

La morphologie spermatique a été ainsi évaluée sur le moniteur avec un grossissement compris entre 3000 et 10225 fois 
Tableau 1: Caractéristiques spermatiques des patients du Groupe 1.

\begin{tabular}{cccccccccccc}
\hline PATIENT & AGE & ABS & VOL & PH & MOB1 & VIT & NUM & FT1 & MACR & IAM & NSMI \\
\hline 1 & 48 & 4 & 4,7 & 7,9 & 16 & 31 & 98,7 & 0 & 96 & 3,35 & 0 \\
\hline 2 & 39 & 4 & 2,5 & 8,2 & 10 & 8 & 42,5 & 0 & 94 & 2,44 & 0,1725 \\
\hline
\end{tabular}

Age en année ; Abs : Abstinence en jours ; Vol: Volume de l'éjaculat en ml; Mob1: mobilité $(a+b+c)$ en \% sur sperme entier ; Vit : vitalité en \% sur sperme entier ; Num : numération en millions/éjaculat sur sperme entier ; FT1: Formes typiques sur sperme entier en \%, IAM : Index d'anomalies multiples ; NSMI : nombre de spermatozoïdes mobiles inséminables en millions/éjaculat.

Tableau 2 : Caractéristiques spermatiques des patients du Groupe 2.

\begin{tabular}{|c|c|c|c|c|c|c|c|c|c|c|c|}
\hline PATIENT & ANOM CHROMO & AGE & ABS & VOL & PH & MOB1 & VIT & NUM & FT1 & IAM & NSMI \\
\hline 3 & $46, X Y, t(10 ; 13)(q 11 ; q 34)$ & 43 & 10 & 8,6 & 7,9 & 24 & 34 & 292,4 & 4 & 2,35 & 8,6 \\
\hline 4 & $46, X Y, t(5 ; 8)(p 15.1 ; q 22.1)$ & 36 & 3 & 3,5 & 7,7 & 66 & 50 & 26,95 & 2 & 2,02 & 5,19 \\
\hline 5 & $46, \mathrm{XY}, \mathrm{t}(4 ; 11)(\mathrm{p} 15.2 ; \mathrm{q} 21)$ & 28 & 1,5 & 2,8 & 8,2 & 58 & 56 & 0,224 & 10 & 2,2 & 0,004 \\
\hline 6 & $46, X Y, t(5 ; 21)(q 15 ; q 22.2)$ & 30 & 4 & 3 & 7,9 & 40 & 76 & 267 & 13 & 1,32 & 32,4 \\
\hline 7 & $46, X Y, t(8 ; 9)(q 21.1 ; q 22.2)$ & 32 & 6 & 7,2 & 8,2 & 50 & 53 & 165,6 & 10 & 1,97 & 21,6 \\
\hline 8 & $46, X Y, t(6 ; 10)(p 23 . q 11.2)$ & 47 & 3 & 3,2 & 7,9 & 40 & 60 & 60,8 & 7 & 2,23 & 6,144 \\
\hline 9 & $45, X Y, \operatorname{rob}(13 ; 14)(q 10 ; q 10)$ & 39 & 3 & 1,4 & 8,2 & 50 & 55 & 5,6 & 12 & 2,39 & 0,8 \\
\hline 10 & $45, \mathrm{XY}, \mathrm{rob}(13 ; 14)(\mathrm{q} 10 ; q 10)$ & 32 & 4 & 4,7 & 7,9 & 30 & 60 & 3,29 & 1 & 1,5 & 0,51 \\
\hline 11 & $45, X Y, \operatorname{rob}(13 ; 14)(q 10 ; q 10)$ & 33 & 3 & 2,2 & 8,2 & 24 & 48 & 0,352 & 4 & 2,15 & 0,005 \\
\hline 12 & $45, \mathrm{XY}, \operatorname{rob}(15 ; 21)(q 10 ; q 10)$ & 39 & 3 & 2 & 7,9 & 40 & 92 & 34 & 32 & 1,53 & 10,14 \\
\hline 13 & $45, X Y, r o b(13 ; 15)(q 10 ; q 10)$ & 25 & 4 & 2,8 & 7,9 & 32 & 72 & 16,24 & 6 & 1,76 & 0,5 \\
\hline
\end{tabular}

Anom chromo: Anomalie chromosomique; rob : translocation robertsonnienne; Age en année ; Abs : Abstinence en jours ; Volume : volume de l'éjaculat en $\mathrm{ml}$; Mob1 : mobilité $(a+b+c)$ en \% sur sperme entier ; Vit : vitalité en \% sur sperme entier; Num : numération en millions/éjaculat sur sperme entier ; FT1 : Formes typiques sur sperme entier en \%; IAM : Index d'anomalies multiples ; NSMI : nombre de spermatozö̈des mobiles inséminables en millions/ejaculat.

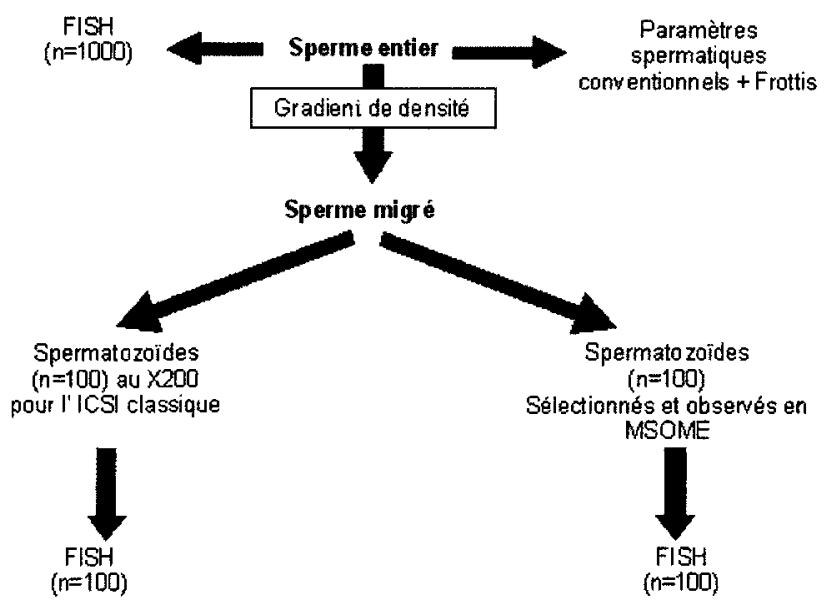

Figure 1 : Protocole d'étude. 
(objectif $\times 100$, caméra/moniteur $\times 30$, vis $\times 1,5$ et zoom compris entre $\times 0,9$ et $\times 2,25$ ). Nous avons utilisé une grille de lecture validée par plusieurs lecteurs, comprenant les critères de la classification morphologique des spermatozoïdes humains selon la méthode de David modifiée [1], à laquelle s'ajoutent la description des vacuoles nucléaires (taille, nombre, surface par rapport à la surface du noyau, localisation) ainsi que deux anomalies de la tête, contours irréguliers, anomalie régionale (invagination ou extrusion) et l'irrégularité de la pièce intermédiaire [35].

Pour chaque patient, les spermatozoïdes sélectionnés en MSOME (Figure 2) en vue d'une lecture en FISH correspondaient à des spermatozoïdes strictement normaux selon les critères de Bartoov, à savoir, un contour régulier, de forme ovale et symétrique, avec un grand axe et un petit axe mesurant respectivement $4,8 \pm$ $0,3 \mu \mathrm{m}$ et $3,3 \pm 0,2 \mu \mathrm{m}$, aucune anomalie et/ou une seule vacuole au niveau de la tête occupant au maximum $4 \%$ de la surface de la tête.

\section{c) FISH sur spermatozoïdes}

Choix des sondes.

Pour tous les patients, nous avons utilisé des sondes centromériques des chromosomes $X$ et $Y$ (CEP $X$ et CEP $Y$, Abbott(C) car les aneuploïdies des gonosomes sont les plus fréquentes chez les enfants issus d'ICSI que dans la population générale [38] et que l'origine des aneuploïdies des gonosomes dans la population générale est aussi bien paternelle que maternelle [15]. Le chromosome 18 a été choisi comme autosome en raison de l'excellente qualité du marquage de la sonde centromérique (CEP 18, Abbott@) sur des noyaux interphasiques.

Dans les cas de remaniement chromosomique, le choix des sondes a été fait en fonction de la translocation concernée, pour nous permettre de distinguer tous les types de ségrégation (Figures 3 et 4).

\section{Préparation des lames pour FISH.}

- FISH sur sperme entier : plusieurs frottis ont été réalisés sur lames dégraissées à partir des spermatozoïdes fixés dans le Carnoy acétique.

- FISH sur spermatozoïdes sélectionnés : nous avons mis au point une méthode de fixation des spermatozoïdes sélectionnés dérivée de celle utilisée pour l'analyse du $1^{\mathrm{er}}$ globule polaire par FISH (dépôt dans une microgoutte d'eau distillée, puis ajout de microgouttes de Carnoy). Les spermatozoïdes, après sélection aux deux différents grossissements ( $x 200$ et $\times 10000)$, ont été déposés dans une microgoutte d'eau distillée sur une lame à puits mise dans une boîte de Pétri pour éviter la déshydratation. A la fin de la sélection, la goutte d'eau est évaporée et les spermatozoïdes sont fixés avec du Carnoy acétique ( 3 volume de méthanol / 1 volume d'acide acétique).

Pour chaque patient, les différentes sélections ont été réalisées sur le même éjaculat, par le même opérateur.

Technique de FISH :
Les lames ont été vieillies 20 min dans une solution de $2 \times S S C$ à $37^{\circ} \mathrm{C}$ puis fixées à nouveau dans du méthanol pendant $5 \mathrm{~min}$. Les noyaux des spermatozoïdes ont été ensuite décondensés dans une solution $1 \mathrm{~N}$ de Soude pendant $1 \mathrm{~min}$ et la lame a été rincée pendant 5 min successivement dans du 2SSC puis du PBS. La lame a été ensuite déshydratée 1 min 30 sec dans des bains successifs d'éthanol à $70 \%, 85 \%$ et $100 \%$. L'hybridation a été réalisée dans un HYBrite $\otimes$ (Vysis) après dépôt dans chaque puit de $3 \mu$ le solution " mix " contenant les sondes. Les préparations ont été codénaturées pendant 4 min à $73^{\circ} \mathrm{C}$, et l'hybridation effectuée à $37^{\circ} \mathrm{C}$ durant 15 heures. Les lames ont été ensuite rincées pendant $1 \mathrm{~min} 45 \mathrm{~s}$ dans une solution $0,4 \mathrm{SSC}, 0,1 \%$ Igepal à $73^{\circ} \mathrm{C}$, puis $15 \mathrm{sec}$ dans une solution 2SSC, $0,3 \%$ Igepal à température ambiante et contre colorées avec $10 \mu \mathrm{l}$ de DAPI. La lecture des lames a été validée en double lecture par 2 opérateurs différents, avec un microscope Olympus $₫$ BX60 équipé d'une lampe à fluorescence et des filtres DAPI, FITC, Rhodamine, Gold et Acqua.

\section{d) Analyse statistique}

Le test du chi 2 (??) a été utilisé afin de comparer chez chaque patient les taux d'aneuploïdies observés sur (1) le sperme entier, (2) les 100 spermatozoïdes sélectionnés selon la méthode classique ICSI, et (3) les 100 spermatozoïdes sélectionnés en MSOME.

\section{RESULTATS}

En moyenne, $85 \%( \pm 10 \%)$ des spermatozoïdes sélectionnés ont pu être analysés par FISH. Cette perte était due aux différents lavages de lame au cours de la technique de FISH.

\section{Patients du groupe 1 (macrocéphalie spermatique)}

Pour les 2 patients présentant le syndrome de macrocéphalie spermatique, le taux d'aneuploïdie spermatique sur sperme frais est supérieur à $99 \%$ (Tableau 3). Pour le patient 1, 31 spermatozoïdes ont été estimés " injectables " après 3 heures d'analyse, et seulement 8 pour le patient 2 (Tableau 3). La sélection en MSOME montre seulement 6 spermatozoïdes trouvés morphologiquement corrects pour le patient 1 et tous étaient haploïdes mais anormaux. Aucun spermatozoïde n'a pu être trouvé pour le patient 2. Nous avons observé une diminution significative de la fréquence de spermatozoïdes polyploïdes entre le sperme entier et la sélection en ICSI $(5,10 \%$ vs $82,30 \%, p=0,0408)$ (Figure 5 ). Ceci s'est accompagné d'une augmentation de la proportion de spermatozoïdes diploïdes $(43,60 \%$ vs $15,05 \%, p<0,0001)$ et haploïdes $(2,65 \%$ vs $51,30 \%, p<0,0001)$. En ce qui concerne la sélection en MSOME, aucune statistique n'a pu être faite.

\section{Les patients ayant une translocation réciproque}

\section{a) Taux d'aneuplö̈die sur sperme entier}

Pour l'analyse de la ségrégation des translocations, en moyenne 1007 spermatozoïdes ont été analysés par patient (entre 213 et 2272 selon les possibilités techniques). Les taux d'aneuploïdie spermatique variaient chez ces patients entre $53,6 \%$ et $32,6 \%$ selon le type d'anomalie (Figure 6). La ségrégation adjacente 1 était, dans 5 cas sur 6 , le principal 

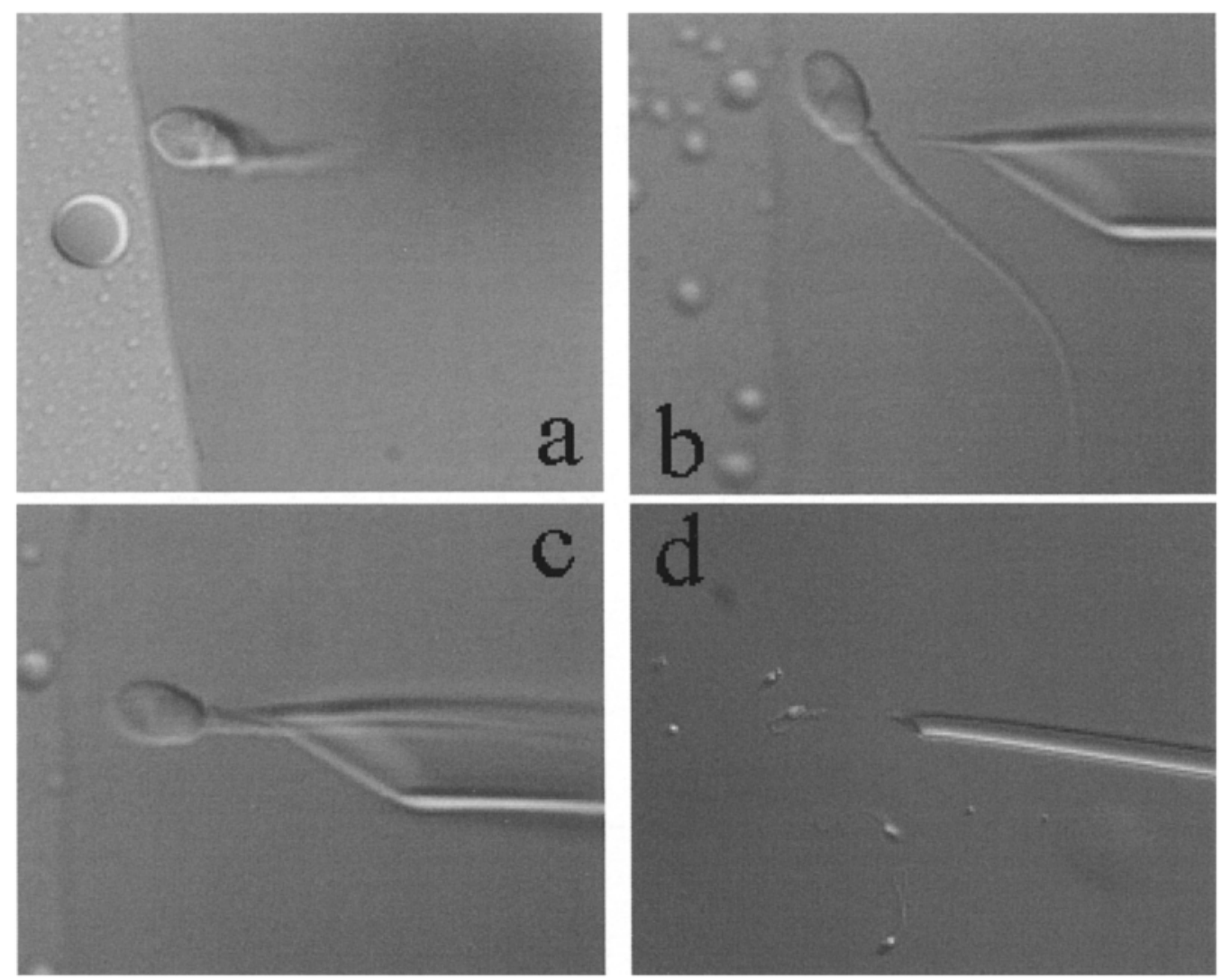

Figure 2 : Etapes successives de la sélection des spermatozoïdes au MSOME.

a : localisation du spermatozoïde en bord de goutte (x6600);

$b$ : mise à plat et observation de la morphologie spermatique (x6600) ;

$c$ : aspiration du spermatozoïde par le flagelle (x6600);

$d$ : dépôt des spermatozoïdes sélectionnés dans une goutte d'eau distillée sur lame (x200).

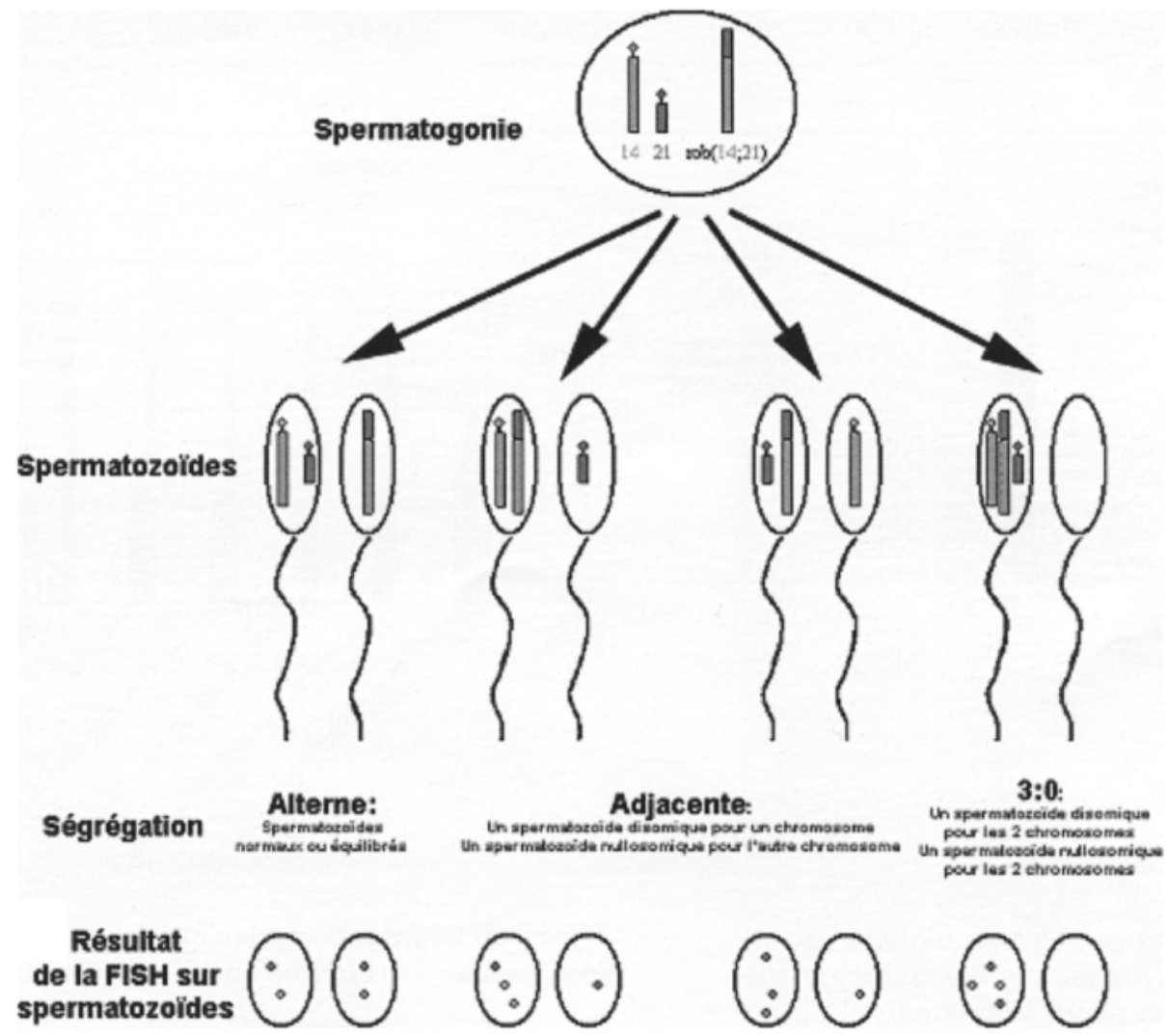

Figure 3 : Ségrégation d'une translocation robertsonnienne. 


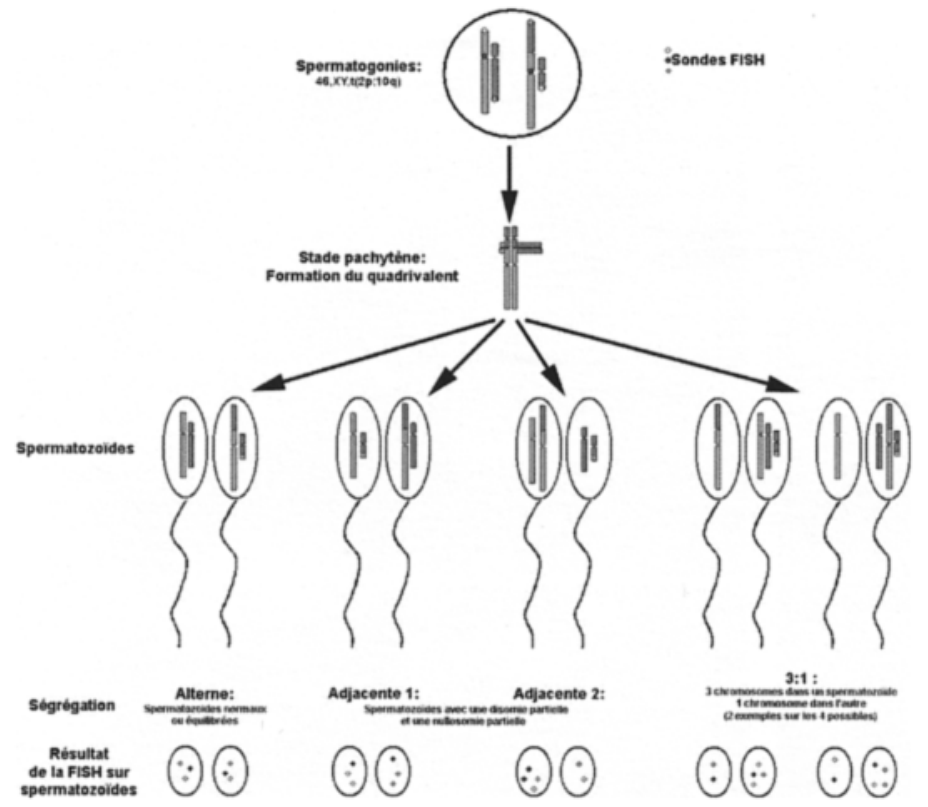

Figure 4 : Ségrégation d'une translocation réciproque et choix des sondes.

Tableau 3 : Taux d'aneuploïdie sur sperme entier et après sélection ICSI chez les patients porteurs d'un syndrome de macrocéphalie spermatique : effectifs et pourcentages.

\begin{tabular}{|c|c|c|c|c|c|c|c|}
\hline PATIENT & $\begin{array}{l}\text { \% MACROCEPHALES } \\
\text { (SPERMOCYTO- } \\
\text { GRAMME) }\end{array}$ & & $\begin{array}{c}\text { FiSH } \\
n=\end{array}$ & $\begin{array}{l}\text { HAPLOIIDES } \\
\text { NORMAUX } \\
\text { n (\%) }\end{array}$ & $\begin{array}{c}\text { HAPLOÏDES } \\
\text { ANEUPLOIDES } \\
n(\%)\end{array}$ & $\begin{array}{l}\text { DIPLOIIDES } \\
\text { n (\%) }\end{array}$ & $\begin{array}{c}\text { TETRAPLOIIDES } \\
n(\%)\end{array}$ \\
\hline \multirow{3}{*}{1} & \multirow{3}{*}{96} & Sperme frais & $\begin{array}{c}101 \\
2 \\
\end{array}$ & $10(0,99 \%)$ & $14(1,38 \%)$ & $\begin{array}{c}200 \\
(19,76 \%) \\
\end{array}$ & $788(77,87 \%)$ \\
\hline & & Sélection ICSI & 31 & $1(3,2 \%)$ & $16(51,6 \%)$ & $14(45,2 \%)$ & $0(0,0 \%)$ \\
\hline & & $\begin{array}{l}\text { Sélection } \\
\text { MSOME }\end{array}$ & 6 & 0 & $6(100 \%)$ & 0 & 0 \\
\hline \multirow{3}{*}{2} & \multirow{3}{*}{94} & Sperme frais & $\begin{array}{c}100 \\
2\end{array}$ & $3(0,30 \%)$ & $26(2,59 \%)$ & $\begin{array}{c}103 \\
(10,28 \%) \\
\end{array}$ & $870(86,83 \%)$ \\
\hline & & Sélection ICSI & 8 & $0(0 \%)$ & $3(37,5 \%)$ & $3(37,5 \%)$ & $2(25,0 \%)$ \\
\hline & & $\begin{array}{l}\text { Sélection } \\
\text { MSOME }\end{array}$ & 0 & 0 & 0 & 0 & 0 \\
\hline
\end{tabular}

$n=$ nombre de spermatozoïdes.
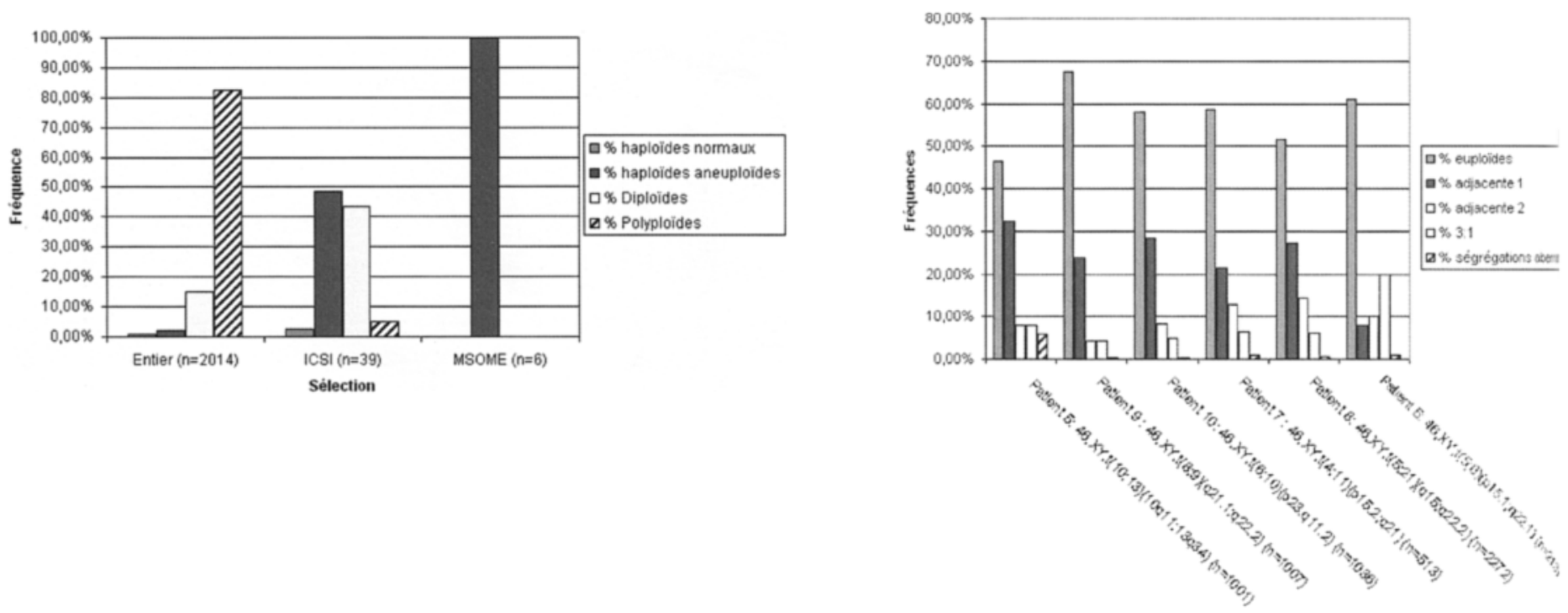

Figure 5 : Analyse comparative des aneuplö̈dies après sélection chez les patients présentant la macrocéphalie spermatique. $n=$ nombre de spermatozö̈des.

Figure 6 : Ségrégations des différentes translocations réciproques sur sperme entier. 
mécanisme à l'origine des aneuploïdies spermatiques ; dans le dernier cas (Patient 6), il s'agissait d'une malségrégation de type 3:1.

Des similitudes existant entre les ségrégations de différents patients, nous avons défini 3 sous groupes de patients en fonction de leur profil de ségrégation (Tableau 4). Les résultats obtenus dans chaque sous-groupe ont été cumulés et sont reportés dans la Figure 7 .

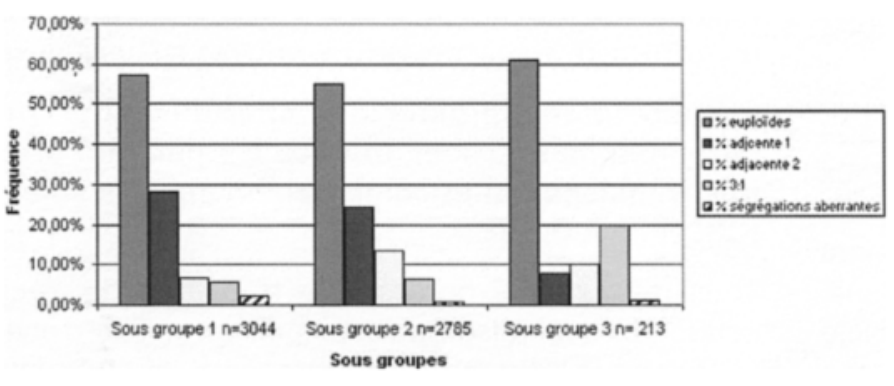

Figure 7 : Analyse du contenu chromosomique des spermatozoïdes sur sperme entier des patients porteurs de translocations réciproques répartis en sous groupes "homogènes". n = nombre de spermatozoïdes.

Au total, les résultats des patients du sous groupe 1 montraient un taux global de spermatozoïdes euploïdes de $57,27 \%$, et les ségrégations adjacente 1, adjacente 2 et 3 : 1 représentaient respectivement $28,23 \%, 6,77 \%$ et $5,70 \%$. Pour le sous groupe 2, le taux de spermatozoïdes euploïdes était de $55,10 \%$ et les ségrégations adjacente 1 , adjacente 2 et 3 : 1 représentaient respectivement $24,34 \%, 13,59 \%$ et $6,29 \%$. Pour le dernier patient, le taux de spermatozoïdes euploïdes était de $61,00 \%$ et les ségrégations adjacente 1 , adjacente 2 et 3 : 1 représentaient respectivement $8,00 \%$, $10,00 \%$ et $20,00 \%$.

L'étude des chromosomes $X, Y$ et 18 a été réalisée pour 5 patients. 514 à 1038 spermatozoïdes par patient ont été analysés, soit une moyenne de 923 spermatozoïdes par individu. Le taux d'aneuploïdie pour les chromosomes $X, Y$ et 18 variait entre 3,76 et $0,49 \%$ (moyenne $1,90 \pm 1,66 \%$ ). Les taux moyens de monosomie et de disomie étaient respectivement de $0,29 \%$ et de $1,49 \%$.

\section{b) Comparaison des taux d'aneuploïdie entre la sélection en ICSI et en MSOME}

Dans le sous groupe 1 (translocation réciproque équilibrée, taux de ségrégation adjacente 1 supérieur à $20 \%$, adjacente 2 et $3: 1$ inférieures à $10 \%$ dans le sperme entier), la sélection en ICSI a permis d'analyser 74 à 153 spermatozoïdes pour chaque patient (105 en moyenne par individu). Les taux d'aneuploïdie spermatique variaient entre 52,7 et $32,2 \%$ (moyenne $36,62 \%$ ). La ségrégation adjacente 1 était toujours le principal mécanisme à l'origine des aneuploïdies spermatiques (moyenne $20,38 \%$ ) et la ségrégation adjacente 2 toujours inférieure à $10 \%$ (moyenne $2,87 \%$ ).

En sélection MSOME, 18 à 132 spermatozoïdes ont été analysés par patient (70 en moyenne). Les taux d'aneuploïdie spermatique variaient entre $77,27 \%$ et $22,00 \%$ (moyenne $37,62 \%$ ). La ségrégation adjacente 1 était toujours le principal mécanisme à l'origine des aneuploïdies spermatiques (moyenne 21,90\%) et la ségrégation adjacente 2 dans tous les cas inférieure à $10 \%$ (moyenne 1,43\%). Nous avons observé une augmentation significative du taux de spermatozoïdes euploïdes entre le sperme entier et l'ICSI $(57,27 \%$ vs $63,36 \%, p=0,0424)$. Ceci s'est accompagné d'une diminution significative de la fréquence des ségrégations adjacente 1 et adjacente 2 et d'une augmentation de celle des 3:1. En comparant le sperme entier et le MSOME, nous avons également observé une augmentation significative du taux de spermatozoïdes euploïdes $(57,27 \%$ vs $62,38 \%, p=0,043)$, associée à une diminution significative de la proportion des ségrégations adjacente $2(p=0,004)$ et d'une augmentation de la 3:1 $(p=0,002)$. Aucune variation significative n'a été observée entre les sélections en ICSI et en MSOME.

Dans le sous groupe 2 (translocation équilibrée, taux de ségrégation adjacente 1 supérieur à $20 \%$ et adjacente 2 supérieur à $10 \%$ sur sperme entier) la sélection en ICSI a permis d'étudier la ségrégation de la translocation sur 140 à 148 spermatozoïdes, soit 144 en moyenne par patient. Le taux d'aneuploïdie spermatique était de $50 \%$. La fréquence de la ségrégation adjacente 1 était toujours le principal mécanisme à l'origine des aneuploïdies spermatiques (moyenne $26,74 \%$ ) et la ségrégation adjacente 2 toujours supérieure à $10 \%$ (moyenne 16,76\%). La ségrégation 3 :1 représentait 5,90\% des ségrégations.

La sélection en MSOME a permis d'analyser la ségrégation de la translocation sur 113 et 73 spermatozoïdes pour les patients 6 et 7 , soit 93 en moyenne par patient. Le taux de spermatozoïdes euploïdes était de $52,69 \%$. La ségrégation adjacente 1 était toujours le principal mécanisme à l'origine des aneuploïdies spermatiques (moyenne 21,51\%). Par contre, la ségrégation adjacente 2 ne représentait plus que $5,38 \%$ des ségrégations et la ségrégation $3: 1$ représentait $13,98 \%$ des ségrégations. Nous n'avons pas observé de variation significative du taux de spermatozoïdes euploïdes, ni de ceux porteurs de la ségrégation adjacente 1. En MSOME,

Tableau 4 : Définition des sous groupes de patients porteurs de translocations réciproques.

\begin{tabular}{|c|c|c|c|c|}
\hline SOUS GROUPE & $\mathbf{N}^{\circ}$ DES PATIENTS & $\%$ ADJACENTE 1 & $\%$ ADJACENTE 2 & $\% 3: 1$ \\
\hline 1 & $5-9-10$ & $>20 \%$ & $<10 \%$ & $<10 \%$ \\
\hline 2 & $7-8$ & $>20 \%$ & $>10 \%$ & $<10 \%$ \\
\hline 3 & 6 & $<10 \%$ & $10 \%$ & $>10 \%$ \\
\hline
\end{tabular}


nous avons observé une diminution de la fréquence de la ségrégation adjacente $2(p<0,002)$ et une augmentation de celle de la ségrégation $3: 1 \quad(p<0,05)$ et des autres configurations $(p<0,0001)$.

Enfin pour le sous groupe 3 (translocation réciproque avec un taux de ségrégation $3: 1$ supérieur à $10 \%$ ) la sélection en ICSI a permis d'analyser la ségrégation de la translocation sur 152 spermatozoïdes. Le taux d'aneuploïdie spermatique était de $53,50 \%$. Les ségrégations adjacente 1, adjacente 2 et $3: 1$ représentaient respectivement $17,11 \%, 5,26 \%$ et $16,45 \%$ des ségrégations.

En sélection MSOME, l'analyse de la ségrégation de la translocation a été réalisée sur 96 spermatozoïdes. Le taux de spermatozoïdes aneuploïdes était de $47,92 \%$. Les ségrégations adjacente 1 , adjacente 2 et $3: 1$ représentaient respectivement $16,67 \%, 0,00 \%$ et $21,88 \%$ des ségrégations. Nous n'avons pas observé de variations du taux d'aneuploïdie entre les différentes techniques. Par contre, nous avons observé une diminution de la fréquence de la ségrégation adjacente 2 en MSOME par rapport au sperme entier $(p=0,0032)$ et une augmentation de la fréquence des ségrégations aberrantes $(p<0,0001)$. Entre les sélections en $|C S|$ et en MSOME, aucune différence significative n'a été retrouvée, exception faite des configurations aberrantes $(p=0,0407)$.

Les résultats de tous les patients porteurs d'une translocation réciproque et répartis dans les 3 sous groupes paraissant voisins, nous les avons regroupés sur la Figure 8. Aucune variation du taux d'aneuploïdie spermatique entre les différentes techniques n'a été observée. Par contre, nous avons observé une diminution significative de la fréquence de la ségrégation adjacente 1 en ICSI et en MSOME par rapport au sperme entier $(p=0,0319$ et $p=0,0138)$, une diminution significative de la fréquence de la ségrégation adjacente 2 en MSOME par rapport au sperme entier $(p<0,0001)$ et l'ICSI $(p<0,0001)$. La fréquence de la ségrégation $3: 1$ a augmenté entre la sélection ICSI et le sperme entier $(p<0,0001)$ ainsi qu'entre le MSOME et l'ICSI $(p<0,0001)$.

\section{Patients ayant une translocation robertsonienne}

\section{a) Taux d'aneuploïdie sur sperme entier}

Concernant les déséquilibres liés à la ségrégation de la translocation, 956 spermatozoïdes en moyenne par patient ont été analysés (entre 689 et 1032). Les taux d'aneuploïdies étaient globalement identiques (entre 28,7 et $20,0 \%$ ) pour les 5 patients concernés.

Pour les chromosomes $X, Y$ et 18,529 à 1170 spermatozoïdes ont été analysés par patient (962 en moyenne). Le taux d'anomalie variait entre 3,97 et $1,82 \%$ (moyenne $2,27 \pm$ $0,95 \%$ ). Les taux moyens de monosomie et de disomie étaient respectivement de $0,88 \%$ et de $1,32 \%$. Comme pour les patients porteurs de translocations réciproques, ces taux se sont révélés comparables pour les patients de ce groupe.

\section{b) Comparaison des taux d'aneuploïdie entre la sélection en ICSI et en MSOME}

La sélection en ICSI a permis d'étudier la ségrégation de la translocation sur 56 à 138 spermatozoïdes (83 en moyenne par patient). Les taux d'aneuploïdie spermatique variaient entre $51,50 \%$ et $20,00 \%$ (moyenne $29,85 \%$ ) avec $29,00 \%$ de ségrégation adjacente. La sélection en MSOME a permis d'analyser 33 à 137 spermatozoïdes (65 en moyenne par patient). Les taux d'aneuploïdie spermatique variaient entre 54,55 et $22,73 \%$. (moyenne $38,38 \%$ ) avec $32,77 \%$ de ségrégation adjacente. Ces différents résultats sont représentés sur la Figure 9. Nous avons observé une augmentation légèrement significative du taux d'aneuploïdie entre le sperme entier et les deux autres techniques $(p<0,02)$.

4. Taux d'aneuploïdie sur sperme entier pour les chromosomes $X, Y$ et 18 chez les patients présentant une translocation et comparaison entre la sélection en ICSI et en MSOME

Tous les patients porteurs de translocation, réciproque équilibrée ou robertsonienne, présentent un taux d'aneuploïdie spermatique pour les chromosomes $X, Y$ et 18 inférieur à $5 \%$ sur sperme entier. La comparaison individuelle de ce taux pour chaque patient porteur de translocation entre les différentes sélections n'a pas montré de variation significative. Nous avons donc choisi de regrouper dans un seul et unique groupe l'ensemble de ces patients et de montrer les résultats cumulés pour les chromosomes $X, Y$ et 18 (Figure 10). Au total, le taux d'aneuploïdie sur sperme entier était de 1,86\% avec $0,53 \%$ de monosomie et $1,32 \%$ de disomie. Pour les spermatozoïdes sélectionnés pour l'ICSI, le taux d'aneuploïdie était de $2,61 \%$ avec $1,21 \%$ de monosomie et $1,40 \%$ de disomie. Au niveau des spermatozoïdes sélectionnés en MSOME, le taux d'aneuploïdie était de $1,67 \%$ avec $0,60 \%$ de monosomie et $1,07 \%$ de disomie. II n'existait aucune différence significative entre les trois techniques.

\section{DISCUSSION}

Notre objectif a été d'évaluer l'intérêt de sélectionner les spermatozoïdes au fort grossissement dans le cadre de la prise en charge de couples à risque d'aneuploïdie augmenté et de savoir si cette technique permettrait de sélectionner des spermatozoïdes ayant de plus grandes chances d'être euploïdes. Plusieurs auteurs se sont déjà intéressés à la relation existant entre la morphologie des spermatozoïdes et leur contenu chromosomique par FISH, chez des patients infertiles à caryotype normal. Le taux d'aneuploïdie spermatique est augmenté chez les patients présentant certaines tératozoospermies observées en microscopie conventionnelle $[23,29]$, mais aussi en microscopie électronique [28]. Cependant, aucune association significative entre la morphologie des spermatozoïdes en microscopie conventionnelle et la fréquence des aneuploïdies chromosomiques spermatiques n'a été actuellement retrouvée $[11,36]$. Nous avons voulu savoir si l'observation de spermatozoïdes sélectionnés pour l'ICSI ou en MSOME pourrait permettre d'améliorer les taux d'aneuploïdie spermatique chez des patients ayant un fort taux d'aneuploïdie spermatique. Nous avons pour cela choisi de travailler sur 2 groupes de patients : groupe 1 : des patients à caryotype normal, ayant une macrocéphalie spermatique ; et groupe 2 : des patients porteurs d'une anomalie chromosomique 


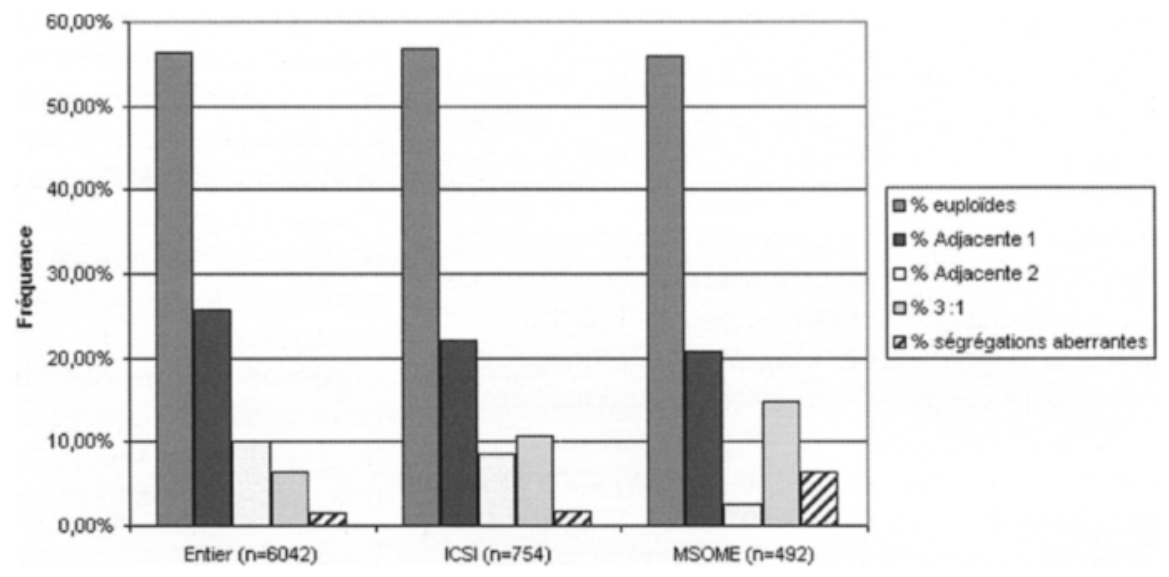

Figure 8 : Proportion des différentes ségrégation pour les patients porteurs d'une translocation réciproque en fonction des différentes techniques. $n=$ nombre de spermatozoïdes.

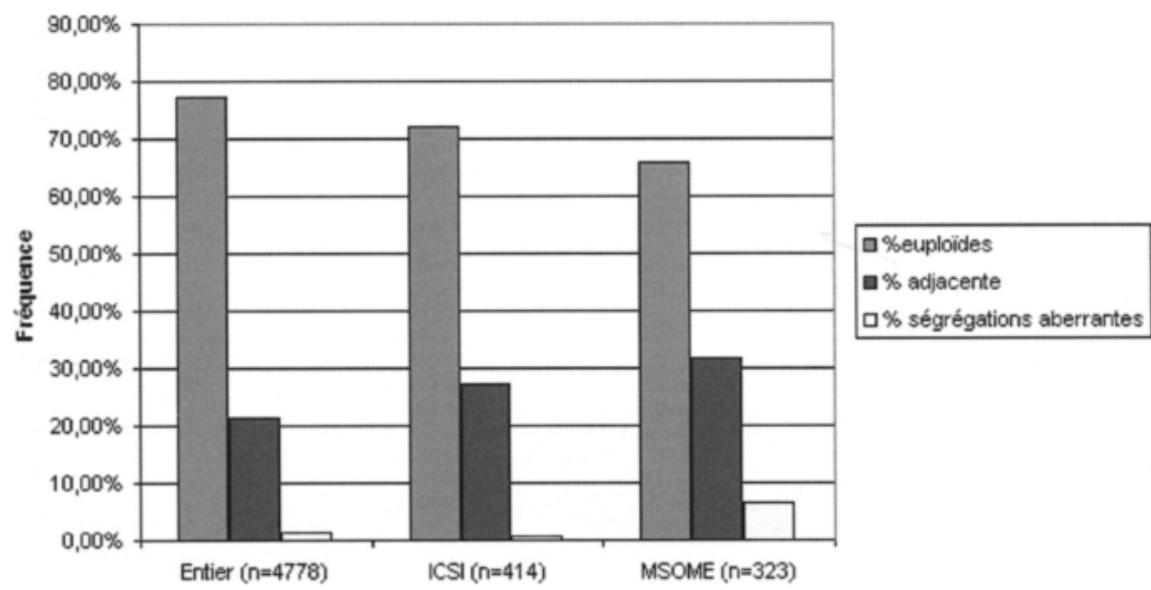

Figure 9 : Taux de ségrégation en fonction de la techniques chez les patients ayant une translocation robertsonnienne. $n=$ nombre de spermatozoïdes.

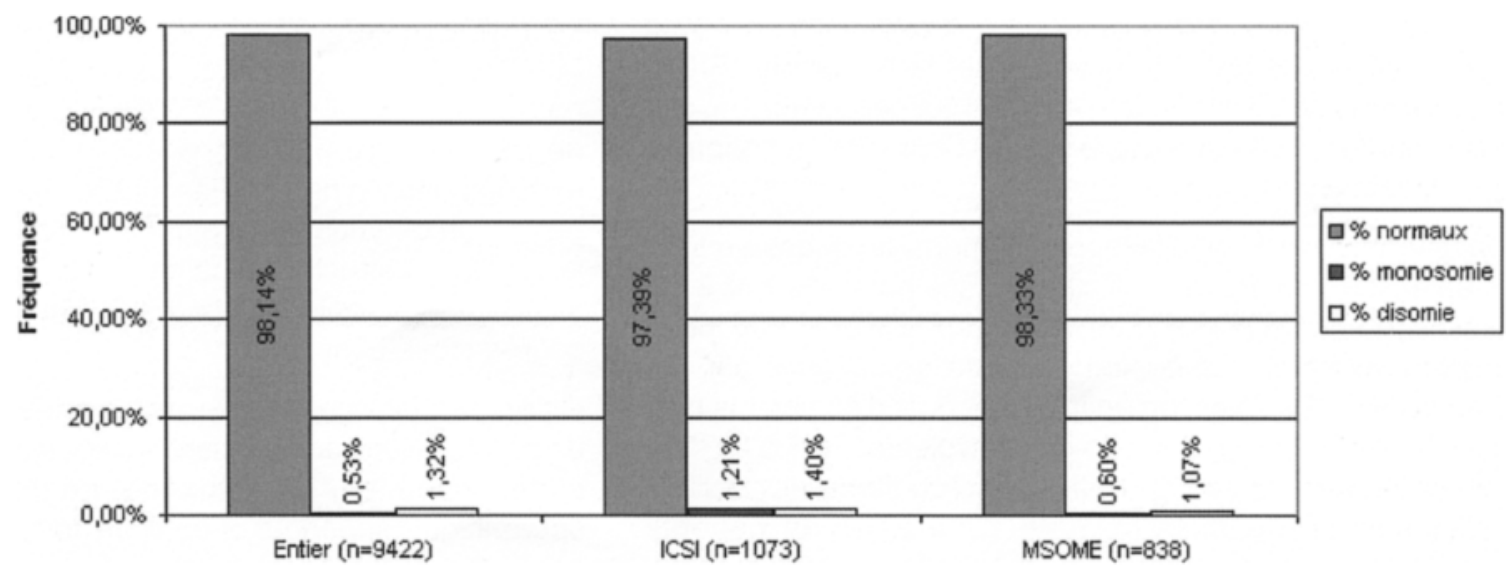

Figure 10 : Taux d'aneuplö̈die des chromosomes $X, Y$ et 18 chez les patients porteurs d'une translocation. $n=$ nombre de spermatozoïdes. 
(translocations réciproques équilibrées et robertsonniennes).

Nous avons effectué, pour chaque patient et sur le même éjaculât, une étude en FISH sur sperme entier, puis sur des spermatozoïdes sélectionnés pour l'ICSI, enfin sur des spermatozoïdes sélectionnés en MSOME, et nous avons comparé les taux d'aneuploïdie entre les trois techniques. La principale difficulté a été la sélection des spermatozoïdes en MSOME, puis la fixation sur lames pour la FISH de ces quelques spermatozoïdes. Ceci était particulièrement vrai chez les patients présentant une oligotératozoospermie majeure (<100 spermatozoïdes migrés et moins de $5 \%$ de formes typiques sur les spermatozoïdes migrés) comme par exemple pour le patient 1 : 6 spermatozoïdes sélectionnés, le patient 2 : aucun spermatozoïde sélectionné et le patient 3 : 18 spermatozoïdes sélectionnés.

Dans la littérature, la plupart des études du contenu chromosomique des spermatozoïdes en FISH rapportent des taux d'aneuploïdie spermatique évalués sur plusieurs milliers de spermatozoïdes, mais concernent des situations où le risque d'aneuploïdie est très faible $(<1 \%)$. Dans notre étude, il s'agissait de patients présentant des pathologies spermatiques parfois sévères (spermatozoïdes peu nombreux et/ou atypiques, donc non conformes à la sélection en MSOME, voire même en ICSI) et des situations à risque chromosomique élevé du fait d'une anomalie du caryotype constitutionnel. Pour toutes ces raisons, le nombre de spermatozoïdes étudiés était parfois faible, voire très faible, mais les résultats restaient informatifs, compte tenu des taux élevés d'anomalies décelés en FISH (entre 20 et $45 \%$ de spermatozoïdes porteurs de déséquilibres chromosomiques pour les patients porteurs de translocations réciproques dans notre étude).

Concernant la fixation des spermatozoïdes, il a été nécessaire de mettre au point une technique adaptée à ces effectifs, parfois très faibles, de spermatozoïdes à étudier en FISH. La fixation a consisté à coller sur lame dégraissée les spermatozoïdes sélectionnés isolément, technique dérivée directement de celle mise au point pour l'étude du $1^{\mathrm{er}}$ globule polaire dans le cadre du diagnostic pré-conceptionnel [39]. Néanmoins, il a subsisté une perte moyenne de $15 \%$ de spermatozoïdes entre la sélection et la lecture en FISH. L'autre difficulté était la lecture en FISH sur cellule unique, que nous avons validée en double lecture pour chaque patient et pour chaque sélection.

Dans le groupe 1, la sélection des spermatozoïdes en ICSI et en MSOME a été très difficile pour les patients 1 et 2 qui présentaient un syndrome de macrocéphalie spermatique et aucun spermatozoïde euploïde n'a été sélectionné par ces 2 approches. La sélection en ICSI a permis d'éliminer la plus grande partie des spermatozoïdes polyploïdes (4,9\% en ICSI versus $82,35 \%$ sur sperme entier). Ceci confirme les résultats précédemment publiés sur une autre série de patients présentant un syndrome de macrocéphalie spermatique [16]. La sélection en MSOME n'a permis de retrouver que 6 spermatozoïdes, tous haploïdes mais aneuploïdes. Les effectifs sont bien sûr très faibles et une conclusion définitive ne peut être apportée, mais néanmoins, on peut noter qu'aucun spermatozoïde sélectionné n'était diploïde et que la sélection en MSOME a finalement permis d'éliminer cette anomalie chromosomique qui à minima est associée à une légère dilatation de la tête spermatique visible au MSOME. Ces résultats confirment qu'en fin de compte, la présence de spermatozoïdes de forme normale dans un syndrome de macrocéphalie pure n'est pas une condition suffisante pour proposer l'ICSI ou I'IMSI aux couples car elle ne garantit en aucun cas le contenu chromosomique normal. Le risque chromosomique pour ces spermatozoïdes reste majeur, pouvant être théoriquement de $100 \%$ si on testait tous les chromosomes et non pas uniquement les chromosomes $X, Y$ et 18.

Le groupe 2 était constitué de patients ayant un caryotype anormal : 6 patients portant une translocation réciproque équilibrée et 5 patients porteurs d'une translocation robertsonnienne. La sélection en ICSI et en MSOME a été possible pour tous les patients, mais encore une fois plus difficile pour les oligotératozoospermies sévères (pour 4 patients, le nombre de spermatozoïdes sélectionnés en MSOME est inférieur à 50). Nous avons tout de même considéré que la lecture en FISH sur un nombre réduit de spermatozoïdes sélectionnés était interprétable vu le risque chromosomique élevé chez ces patients. On sait que l'effet des translocations sur la spermatogenèse est variable d'une translocation à une autre [31] et, pour une même translocation, d'un individu à un autre [24]. On ne pouvait donc pas affirmer, à priori, que l'effet de la sélection en MSOME serait le même pour toutes les translocations. Pour éviter l'effet patient pouvant être lié à une translocation spécifique, nous avons classé nos patients en sous groupes, puis finalement nous les avons cumulés, car effectivement les mêmes conclusions s'appliquaient à tous nos patients. Selon notre étude et sur cette petite série de patients, la sélection des spermatozoïdes en ICSI et en MSOME ne permet pas d'éliminer les spermatozoïdes porteurs de translocation déséquilibrée.

Pour les patients ayant une translocation réciproque et quels que soient les sous groupes définis préalablement selon les différents profils de ségrégation, les taux globaux d'aneuploïdie spermatique ne variaient pas après sélection en ICSI ou en MSOME. Concernant les types de ségrégation, nous avons noté que la sélection en MSOME semble diminuer la proportion de déséquilibre par ségrégations adjacente 1 et 2 . Ces malségrégations ne modifiant pas le nombre mais la structure des chromosomes, pourraient être visibles en MSOME, par action sur la structure et la localisation de la chromatine au sein du noyau et expliquer les variations de fréquences de ségrégations observées.

Or, il a été établi que les spermatozoïdes des patients porteurs de translocations présentaient un taux d'apoptose spermatique matérialisée par un taux de fragmentation de I'ADN spermatique supérieur à celle de témoins [8]. La variation des fréquences de ségrégations retrouvée dans notre étude pourrait suggérer que l'apoptose des spermatozoïdes porteurs de certains déséquilibres (adjacente 1 et adjacente 2) serait plus précoce pour les spermatozoïdes de morphologie nucléaire altérée alors qu'elle serait moins rapide dans les 
spermatozoïdes de morphologie nucléaire à priori normale. Il a été établi que les chromosomes s'organisent en territoires au niveau des noyaux cellulaires [7]. Ces ségrégations pourraient faire partie des causes d'altérations de la localisation nucléaire des chromosomes spermatiques, conduisant à une probable désorganisation de la structure de la chromatine et à l'expression de certains gènes dont ceux régulant l'apoptose [19]. Ces résultats sont, bien sûr, à considérer avec précaution étant donné la petite taille de nos effectifs.

Pour les patients ayant une translocation robertsonnienne, les taux d'aneuploïdie spermatique ne paraissent pas diminués par la sélection et paraissent même augmentés. Encore une fois, la faiblesse des effectifs ne permet pas de conclure à une altération du contenu chromosomique avec la sélection en ICSI ou en MSOME, mais confirme, en tout état de cause, que ces procédés de sélection n'améliorent pas les taux d'aneuploïdies concernant un chromosome entier, qu'il s'agisse d'un chromosome acrocentrique ou d'un gonosome.

La différence dans les variations du taux et du type de malségrégation que nous avons constatée entre les translocations réciproques et les translocations robertsonniennes pose question. Une hypothèse serait que la présence d'un chromosome en plus ou en moins dans son intégralité dans les translocations robertsonniennes ou les aneuploïdies concernant les chromosomes $X, Y$ et 18 ne modifierait pas la morphologie du noyau tel qu'il peut être visualisé en MSOME et que ces aneuploïdies ne sont de ce fait pas éliminées. A l'inverse, les altérations de la structure des chromosomes induites par les cassures des translocations réciproques pourraient s'associer à des modifications de la compaction du noyau (chromatine, histone, fragmentation de l'ADN ou autres ?) ce qui aurait un impact sur l'aspect morphologique du noyau en MSOME, d'où leur élimination partielle lors de la sélection.

Ainsi, pour les patients à risque chromosomique augmenté que nous avons étudiés, la sélection en ICSI ou en MSOME ne permet pas de sélectionner les spermatozoïdes ayant un contenu chromosomique normal. Elle pourrait cependant éliminer partiellement certaines aberrations de la structure chromosomique. Or, la sélection des spermatozoïdes au MSOME semble améliorer le taux d'implantation et de grossesse [18]. Cette sélection est principalement basée sur l'élimination des spermatozoïdes ayant de larges vacuoles [2, 3] qui semblent être d'origine nucléaire. Les ségrégations adjacentes 1 et 2 pourraient entraîner des anomalies morphologiques de ce type. De plus, une corrélation entre le taux de spermatozoïdes à $A D N$ fragmenté et le taux de spermatozoïdes de morphologie fine globale anormale a été démontrée $[4,5,6,9]$ et il semblerait que l'apparition de ces vacuoles soit associée à une augmentation de la fragmentation de l'ADN par une action enzymatique dont l'activité optimale est à la température de $37^{\circ} \mathrm{C}[26]$.

Au total, nous avions émis l'hypothèse que le MSOME permettrait une élimination des spermatozoïdes aneuploïdes. Nos résultats montrent que la sélection en MSOME permet l'élimination des spermatozoïdes diploïdes dont la taille est probablement légèrement augmentée, mais ne permet pas d'éliminer les spermatozoïdes haploïdes aneuploïdes, que le patient présente une tératozoospermie majeure (macrocéphalie) ou qu'il soit porteur d'une translocation. Seuls certains types de ségrégations semblent être sélectionnés par le MSOME. L'amélioration des résultats en terme de grossesse obtenue en utilisant la sélection en MSOME, n'est donc probablement pas due à l'élimination des spermatozoïdes aneuploïdes, mais à la sélection de spermatozoïdes de " meilleure structure nucléaire ».

\section{RÉFÉRENCES}

1. AUGER J., EUSTACHE F. : Standardisation de la classification morphologique des spermatozoïdes humains selon la méthode de David modifiée. Andrologie, 2000, 10 : 358-373.

2. BARTOOV B., BERKOVITZ A., ELTES F., KOGOSOWSKY A., MENEZO Y., BARAK Y. : Real-time fine morphology of motile human sperm cells is associated with IVF-ICSI outcome. J. Androl., 2002, $23: 1-8$.

3. BARTOOV B., BERKOVITZA., ELTES F. et al. : Pregnancy rates are higher with intracytoplasmic morphologically, selected sperm injection than with conventional intracytoplasmic injection. Fertil. Steril., 2003, $80: 1413-1419$.

4. BERKOVITZ A., ELTES F., YAARI S. et al. : The morphological normalcy of the sperm nucleus and pregnancy rate of intracytoplasmic injection with morphologically selected sperm. Hum. Reprod., 2005, $20: 185-190$.

5. BERKOVITZA., ELTES F., ELLEBOGEN A., PEER S., FELDBERG D., BARTOOV B. : Does the presence of nuclear vacuoles in human sperm selected for ICSI affect pregnancy outcome ? Hum. Reprod., 2006, $21: 1787-1790$.

6. BERKOVITZ A., ELTES F., LEDERMAN H. et al. : How to improve IVF-ICSI outcome by sperm selection. RBM Online, 2006, $12: 634$ 638.

7. BRANCO M.R., POMBO A. : Intermingling of chromosome territories in interphase suggests role in translocations and transcriptiondependent associations. Plos. Biol., 2006, 4 : 780-788.

8. BRUGNON F., VAN ASSCHE E., VERHEYEN G. et al. : Study of markers of apoptosis and meiotic segregation in ejaculated sperm of chromosomal translocation carrier patients. Hum. Reprod., 2006, $21: 685-693$.

9. BOUGHALI H., WITTEMER C., VIVILLE S. : Intérêt de l'analyse de la morphologie fine et de la qualité nucléaire des spermatozoïdes dans le cadre des techniques d'AMP. Andrologie, 2006, $16: 38-45$.

10. CALOGERO A.E., BURRELLO N., DE PALMA A., BARONE N., D'AGATA R., VICARI E. : Sperm aneuploidy in infertile men. RBM Online, 2003, $6: 310-317$.

11. CELIK-OZENCI C., JAKAB A., KOVACS T. et al. : Sperm selection for ICSI : shape properties do not predict the absence or presence of numerical chromosomal aberrations. Hum. Reprod., 2004, 19 : 2052-2059.

12. DAVID G., BISSON J.P., CZYGLIK $F$ et al. : Anomalies morphologiques du spermatozoïde humain. Proposition pour un système de classification. J. Gynecol. Obstet. Biol. Reprod., 1975, Suppl.1 : 17-36.

13. DE VOS A., VAN DE VELDE H., JORIS H., VERHEYEN G., DEVROEY P., VAN STEIRTEGHEM P. : Influence of individual sperm morphology on fertilization, embryo morphology, and pregnancy outcome of intracytoplasmic sperm injection. Fertil. Steril., 2003, 79: 42-48.

14. FAURE A.K., AKIN-SEIFER I., FREROT G. et al. : Predictive factors for an increase risk of sperm aneuploidies in oligo-asthenoteratozoospermic males. Int. J. Androl., 2007, 30: 153-162. 
15. GARDNER R., SUTHERLAND G. : Chromosome Abnormalities and Genetic Counselling. 2nd ed., New York, Oxford University Press, 1996.

16. GUTHAUSER B., VIALARD F., DAKOUANE M., IZARD V., ALBERT M., SELVA J. : Chromosomal analysis of spermatozoa with normalsized heads in two infertile patients with macrocephalic sperm head syndrome. Fertil. Steril., 2006, $85: 750$ e5-750 e7.

17. HASSOLD T., HUNT P. : To err (meiotically) is human : the genesis of human aneuploidy. Nat. Rev. Genet., 2001, $2: 280-291$.

18. HAZOUTA., DUMONT-HASSAN M., JUNCAA.M., COHEN BACRIE P., TESARIK J. : High-magnification ICSI overcomes paternal effect resistant to conventional ICSI. RBM Online, 2006, 12 : 19-25.

19. HAZZOURI M., ROUSSEAU S., MONGELARD F. et al. : Genome organization in the human sperm nucleus studied by FISH and confocal microscopy. Mol. Reprod. Dev., 2000, 55 : 307-315.

20. KAHRAMAN S., BENKHALIFA M., DONMEZ E. et al. : The results of aneuploidy screening in 276 couples undergoing assisted reproductive techniques. Prenat. Diagn. 2004, 24 : 307-311.

21. KRUGER, T. F., MENKVELD R., F.S. STANDER F.S. et al. : Sperm morphologic features as a prognostic factor in in vitro fertilization. Fertil. Steril., 1986, 46 : 1118-1123.

22. LIEBAERS I., BONDUELLE M., VAN ASSCHE E., DEVROEY P., VAN STEIRTEGHEM A. : Sex chromosome abnormalities after intracytoplasmic sperm injection. Lancet, 1995, 346: 1095.

23. MACHEV N., GOSSET P., VIVILLE S. : Chromosome abnormalities in sperm from infertile men with normal somatic karyotypes: teratozoospermia. Cytogenet. Genome Res., 2005, $111: 352-357$.

24. MOREL F., ROUX C., BRESSON J.L. : FISH analysis of the chromosomal status of spermatozoa from three men with 45,XY,der(13;14)(q10;q10) karyotype. Mol. Hum. Reprod., 2001, 7: 483-488.

25. PANG M. G., HOEGERMAN S.F., CUTICCHIAA.J. et al. : Detection of aneuploidy for chromosomes $4,6,7,8,9,10,11,12,13,17,18$, $21, X$ and $Y$ by fluorescence in-situ hybridization in spermatozoa from nine patients with oligoasthenoteratozoospermia undergoing intracytoplasmic sperm injection. Hum. Reprod., 1999, 14 : 1266-1273.

26. PEER S., ELTES F., BERKOVITZA. et al. : Is fine morphology of the human nuclei affected by in vitro incubation at $37^{\circ} \mathrm{C}$ ? Fertil. Steril., 2007, $88: 1589-1594$.

27. PETIT F.M., FRYDMAN N., BENKHALIFA M. : Could sperm aneuploidy rate determination be used as a predictive test before intracytoplasmic sperm injection? J. Androl., 2005, 26 : 235-241.

28. PRISANT N., ESCALIER D., SOUFIR J.C. et al. : Ultrastructural nuclear defects and increased chromosome aneuploidies in spermatozoa. Hum. Reprod., 2007, 22 : 1052-1059.

29. RIVES N., SAINT CLAIR A., MASURIER S. et al : Relationship between phenotype, semen parameters and aneuploïdy frequency in sperm nuclei of 50 infertile males. Hum. Genet., 1999, $105: 266-$ 272.

30. RIVES N., MOUSSET-SIMEON N., MAZURIER S., MACE B. : Primary flagellar abnormality is associated with an increased rate of spermatozoa aneuploidy. J. Androl., 2005, 26 : 61-69.

31. ROUSSEAUX S., CHEVRET E., MONTEIL M. et al. : Meiotic segregation in male heterozygote for reciprocal translocations : analysis of sperm nuclei by two and three colour fluorescence in situ hybridization. Cytogenet. Cell. Genet., 1995, 71 : 240-246.

32. RUBIO C., GIL-SALOM M., SIMON C. et al. : Incidence of sperm chromosomal abnormalities in a risk population : relationship with sperm quality and ICSI outcome. Hum. Reprod., 2001, 16 : 2084-2092.

33. SERMON K., MOUTOU C., HARPER J. et al. : ESHRE PGD Consortium data collection IV : May-December 2001. Hum. Reprod., 2005, $20: 19-34$.

34. SHI Q., MARTIN R.H. : Aneuploidy in human spermatozoa: FISH analysis in men with constitutional chromosomal abnormalities, and in infertile men. Reproduction, 2001, $121: 655-666$.
35. SERMONDADE N., VIALARD F., BERGERE M. et al. : Evaluation de l'apport de la methode d'observation des spermatozoïdes à fort grossissement en ICSI. Andrologie, 2007, $17: 212-221$.

36. SUN F., KO E., MARTYIN R.H. : Is there a relationship between sperm chromosome abnormalities and sperm morphology ? Reprod. Biol. Endocrinol., 2006, $25: 4: 1$.

37. TESARIK J., MENDOZA C. : Treatment of severe male infertility by micromanipulation-assisted fertilisation : an update. Front. Biosci., 2007, 12 : 105-114.

38. VAN STEIRTEGHEM A., M. BONDUELLE M., DEVROEY P., LIEBAERS I. : Follow-up of children born after ICSI. Hum. Reprod. Update, 2002, $8: 111-116$.

39. VIALARD F., PETIT C., BERGERE M. et al. : Evidence of high proportion of premature unbalanced separation of sister chromatids in the first polar bodies of women of advanced age. Hum. Reprod., 2006, 21 : 1172-1178.

40. VIVILLE S., MOLLARD R., BACH M.L., FALQUET C., GERLINGER P., WATER S. : Do morphological anomalies reflect chromosomal aneuploidies ? : case report. Hum. Reprod., 2000, 16 : 1779-1780.

Manuscrit reşu : août 2008 ; accepté août 2008.

Prix Master Pro SALF 2007

\section{ABSTRACT}

Can high-power morphological selection of spermatozoa be effective to reduce the chromosomal risk for patients with high rates of sperm aneuploidy?

\section{Mohamed Hassen CHELLI, Martine ALBERT, Denise MOLINA GOMEZ, Ibrahim HAMMOUD, Jaqueline SELVA, François VIALARD}

Severe male infertility concerns two categories of men. Men with abnormal karyotype, who represent 2 to $14 \%$ of infertile men and who can produce sperm cells carrying unbalanced chromosomes related to the patients initial chromosomal reorganization inducing a variable risk of transmission of the abnormality to their conceptus. The second category is men with a normal karyotype but an increased rate of spermatic aneuploidy in a context of severe oligo- and/or asthenozoospermia and men from couples in implantation failure. ICSI is the standard Assisted Medical Reproductive technique for most of these 2 categories despite the obvious increased chromosomal risk. This raises the question of how to morphologically identify sperm cells with abnormal chromosome content during ICSI ?

Unfortunately, no relationship has yet been found between sperm morphology in the ICSI sperm fraction (X200) and their chromosome content. Nevertheless, since the end of the 1990s, Bartoov's team has developed MSOME (Motile Sperm Organelle Morphology Examination) consisting of high-power examination of sperm cells up to $x 12,250$. This technique was indicated for cases of repeated ICSI failures and appeared to increase pregnancy rates. But was this improvement due to better selection of the chromosomal 
content of sperm cells to be injected? The present study addressed this question by estimating the value of MSOME in the selection of euploid sperm cells in 2 groups of patients known to have an increased rate of sperm aneuploidy.

Group 1 was composed of 2 patients with normal karyotype who presented a macrocephalic sperm syndrome with more than $99 \%$ of aneuploid sperm. Group 2 was composed of 11 patients with abnormal karyotype: 6 patients with reciprocal translocation and 5 patients with Robertsonian translocation.

The purpose of this study was to compare spermatozoa aneuploidy rates in fresh semen, to those obtained after ICSI selection (x200) and MSOME selection (x6000). Three specific steps of the protocol were (1) all sperm cells selected in MSOME were "top sperm cells" (2) fixation of selected sperm cell (average loss of $15 \%$ during FISH washes) (3) FISH results were validated by two different examiners.

FISH analysis of $X, Y$ and 18 chromosomes showed that MSOME eliminates polyploid and diploid sperm cells in patients with macrocephalic sperm syndrome, but the 6 sperm cells selected were all haploid and aneuploid.

FISH analysis of $X, Y$ and 18 chromosomes of all other patients did not show any influence of the selection method on the aneuploidy rate. For the 5 subjects with a Robertsonian translocation, the global results of FISH analysis paradoxically showed a significant decrease of the euploidy rate in MSOME selection. The global results of FISH analysis for the 6 patients with mutual reciprocal translocations, showed that the various mutual translocations were not modified between whole sperm and the 2 selection methods. On the other hand, a significant decrease of adjacent 1 and 2 segregation frequency was observed between whole sperm and MSOME selection, associated with a significant increase of $3: 1$ segregation frequency suggesting that the segregations which modify the structure of chromosomes, for example adjacent 1 and 2 segregations, would induce visible morphological modifications selected by MSOME. We hypothesized that the efficacy of spermatic apoptosis could be modulated by morphology but also by the chromosome contents of the sperm cell.

In conclusion, MSOME does not provide any guarantee of the normal chromosome contents of the TOP selected sperm cell. However, these results obtained in a small series of patients suggest that MSOME can eliminate some chromosome abnormalities (adj1 and 2) which would alter sperm nuclear structures.

Key words : spermatozoa, morphology, MSOME, macrocephaly, translocation, FISH 